\title{
Numerical Characterization of Helicopter Noise Hemispheres
}

\author{
M. Gennaretti ${ }^{\mathrm{a}, *}$, G. Bernardini ${ }^{\mathrm{a}}$, J. Serafini ${ }^{\mathrm{a}}$, A. Castorrini ${ }^{\mathrm{b}}$, \\ G. De Matteis ${ }^{\mathrm{b}}$, G. Avanzinic \\ ${ }^{a}$ University Roma Tre, Department of Engineering \\ Via della Vasca Navale 79, 00146 Rome, Italy \\ ${ }^{b}$ University of Rome 'La Sapienza', Dept. of Mechanical and Aerospace Engineering \\ Via Eudossiana 18, 00184 Rome, Italy \\ ${ }^{c}$ Università del Salento, Department of Ingegneria dell'Innovazione \\ 72100 Brindisi, Italy
}

\begin{abstract}
Numerical tools aiming at the evaluation of ground acoustic impact of helicopters typically rely on databases given in terms of acoustic disturbance over hemispheres surrounding the helicopter (noise hemispheres). These are evaluated for a discrete number of steady flights falling within the flight envelope. The objective of the present work is the identification of flight parameters to be considered for the characterization of noise hemispheres, particularly when related to unsteady maneuvers. To this purpose, different approaches based on steady flight aeroelastic/aerodynamic/aeroacoustic predictions are examined for assessing their capability of simulating the acoustic impact of helicopters in arbitrary unsteady flight. The numerical investigation demonstrates that at least three parameters, including disk loading, are required to adequately characterize noise hemispheres. Conversely, the similarity of kinematic parameters alone may yield steady flight acoustic predictions poorly correlated with those obtained for unsteady maneuvers.
\end{abstract}

Keywords: computational aeroacoustics, helicopter noise hemisphere, unsteady maneuver noise, inverse helicopter dynamics, rotor aeroelasticity

\footnotetext{
*Corresponding author. [Tel.: +39 06 57333260; fax: +39 06 5593732]

Email address: massimo.gennaretti@uniroma3.it (M. Gennaretti)
} 


\section{Introduction}

Noise emission represents one of the main drawbacks of civil aviation operations on human communities. In particular, the reduction of the acoustic impact leading to a wider public acceptance of helicopter support to human activities in populated areas is among the present and near-future strategic goals of rotorcraft operators. This interest is demonstrated by EU-funded large research efforts in the framework of the current Green Rotorcraft project within the European Joint Technology Initiative Clean Sky, starting from the results of the former Integrated Project FRIENDCOPTER, that was part of the EU Sixth Framework Program. Past and current research has been exploring several approaches aimed at alleviating helicopter acoustic annoying effects, ranging from (active) higher harmonic control and individual blade control techniques $[1,2,3,4]$ to (passive) innovative design solutions like, for instance, advanced blade tip shape $[5,6,7,8]$.

One of the most relevant contributions to helicopters acoustic impact comes from the noise generated aerodynamically by blade-vortex interactions (BVIs). BVI noise has a specific critical influence on the community acceptance of helicopter civil applications: indeed, being of impulsive nature, it is particularly annoying for the human ear, and typically occurs when the helicopter operates near the ground (in descent or in slow advancing flight) [9]. Among passive techniques aiming at reducing rotorcraft noise footprint, the definition of arrival (near ground) optimal flight trajectories that limit the occurrence of strong BVIs events and acoustic prediction techniques suited to this purpose were investigated in the recent past in several papers $[10,11,12,13,14,15,16,17]$. These works follow approaches similar to those introduced by the fixed-wing aircraft research community involved in the identification of noise abatement flight procedures (see, for instance, Refs. [18, 19, 20]).

Such approaches often combine a flight simulation model, a noise model and a geographic information system to adapt the optimization procedure to the orography and population density distribution of the specific interested area. Noise models used in rotorcraft trajectory optimization tools (and, more generally, for rotorcraft ground acoustic impact evaluation) typically consider near-field acoustic sources, followed by a far-field propagation model that takes into account atmospheric effects (like, for instance, absorption, wind-shear, temperature gradients, humidity) and ground reflection. Acoustic sources are given in terms of sound spectrum distribution over hemispheres 
surrounding the helicopter (the so-called noise hemispheres), featuring acoustic effects of flight in terms of both intensity and directivity of disturbance. During the analysis of noise footprints associated to an arbitrary unsteady flight, the acoustic source needs to be updated in accordance with the instantaneous flight condition experienced by the helicopter (the concept is shown in Fig. 1). Observing that maneuver characteristic time is typically longer than that of rotor aeroelastic/aeroacoustic phenomena, this is accomplished through identification of the noise hemisphere within an appropriate database related to steady-state, rectilinear flights of trimmed helicopters (i.e., flights with constant velocity, vehicle attitude and pilot commands). Such approach is applied to avoid the extremely high computational cost required by the direct evaluation of noise hemispheres along the actual unsteady maneuver. The database is obtained either by off-line computations or by measured data $[13,17]$, and it is defined in a domain of flight parameters that represent the acoustic source state. Several tools devoted to the development of low-noise operating procedures of helicopters (or, more generally, to the assessment of their environmental acoustic impact) consider noise hemisphere databases given in terms of only kinematic flight parameters, like airspeed and flight path angle (see, for instance, Refs. [10, 17]).

The scope of the present work is the identification of the flight parameters that properly characterize helicopter noise hemispheres. This represents a crucial issue in modelling noise emitted by maneuvering helicopters on the basis of a finite set of trim-state acoustic sources. For given points on an arbitrary helicopter trajectory related to typical unsteady flight conditions (decelerated descent, coordinated turn, etc.), steady-state, rectilinear flights reproducing subsets of the associated flight parameters (velocity, flight path angle, main rotor shaft angle, etc.) are examined and the corresponding noise hemispheres are evaluated and compared. From this study, it will be possible to identify those flight parameters that need to be matched in order to assure, at a satisfactory level of accuracy, the (instantaneous) noiseemitted equivalence between a maneuver and a steady-state, rectilinear flight. In order to perform the proposed analysis, an unsteady approach maneuver is defined first, and an inverse flight dynamics simulation tool is then applied for determining the time-histories of corresponding pilot commands, hub loads and helicopter attitude variables [21, 22, 23]. Next, three different criteria are applied to correlate steady-state, rectilinear flights with the local specific unsteady flight conditions at the selected trajectory points.

The numerical investigation described above requires the application of 
multidisciplinary tools, ranging from flight dynamics to aeroacoustics, with inclusion of rotor aeroelasticity. For each one of the steady flights considered, the main rotor aeroelastic response is evaluated through a freewake, harmonic-balance, modal formulation, followed by the application of a boundary integral approach to predict the aeroacoustic emissions used to determine the corresponding noise hemisphere [25, 26, 27, 28]. Indeed, in this kind of analysis, the introduction of an aeroelastic solver relying on a free-wake aerodynamic model is essential, in that the accurate prediction of aeroacoustic phenomena related to BVIs requires the accurate evaluation of the relative position between blades and wake vortices (miss distance) [27].

A detailed explanation of the method for analysis of noise hemisphere equivalence is provided in the next section, with numerical results concerning its application to the flight of a lightweight helicopter presented and discussed in Section 3. Concluding remarks are given in Section 4. Appendices A and $\mathrm{B}$ provide outlines on the flight dynamic, aerodynamic, aeroelastic and aeroacoustic formulations applied in the numerical predictions.

\section{Method of Analysis}

Acoustic annoyance perceived by communities during approach flights of helicopters is strongly dependent on the trajectories flown and it is usually dominated by BVI noise. Tools for searching optimal trajectories that minimize acoustic impact will converge, in general, to solutions based on a sequence of unsteady maneuvers, which include turns, variation of speed and descent angles and, consequently, engine and rotor regimes. On the other side, noise hemispheres applied in these tools are based on steady flight acoustic analyses, that is, in flight regimes that may differ significantly from those required for flying the minimum-noise optimal path.

With the aim of determining criteria for identification of steady, rectilinear flight conditions that are acoustically equivalent to unsteady maneuver states, the method of analysis proposed in this work consists of a three-step process.

The first step is the definition of an arbitrary unsteady approach flight path starting from a level, steady flight condition, and ending with a lower altitude, low-velocity final phase of landing prior to touch-down. The maneuver is prescribed in terms of helicopter center of mass trajectory, to be flown at a prescribed velocity and sideslip angle, for a total of four trajectory variables that need to be defined. This flight path becomes the test bed for 
the comparison among three different approaches examined for the determination of the acoustic equivalence criteria between unsteady and steady-state flights.

In the second step, the time history of flight data associated to the prescribed trajectory are determined through application of an inverse simulation algorithm $[21,22,23]$. The inverse flight dynamics tool is implemented for a low order helicopter model. The fuselage is assumed as rigid and its motion is described in terms of center of mass displacement and yaw, pitch and roll attitude angles. Main rotor dynamics is described by means of an individual blade model featuring articulated rigid blades and a three-state, first-order model for the main rotor inflow velocity. Tail rotor is modeled using momentum theory, with a uniform inflow. Given the expected variation of airspeed, and climb, heading and sideslip angles as a function of time, this model allows to efficiently compute pilot control commands (namely, main rotor collective and cyclic pitch, and tail rotor collective pitch) necessary for tracking the prescribed flight path (a nominal problem is solved, with a number of specified variables equal to available controls [21]). Further, it yields additional flight data that characterize the unsteady flight, like helicopter attitude angles, main rotor disc orientation with respect to relative wind, and low-frequency main rotor loads transmitted to the fuselage, that are conveniently used for the next aeroacoustic analysis. Appendix A provides a more detailed description of the flight dynamics inverse simulation tool adopted for the present study, whereas a complete presentation of the algorithm can be found in [23].

The third step consists in the application of different criteria to select the steady conditions of a flight that satisfactorily might simulate the acoustic disturbance generated by the manuvering helicopter, at selected points along the prescribed trajectory. Specifically, starting from the evolution of advance ratio, $\mu$, climb/descent angle, $\gamma$, pilot control commands, $\theta_{0}, A_{1 s}, B_{1 s}$, shaft angle, $\alpha_{s h}$, and hub forces, $F_{x}, F_{y}, F_{z}$, and moments, $M_{x}, M_{y}$, given in a nonrotoating hub frame, three approaches are introduced to estimate the noise emission at specific points along the trajectory, based on three different definitions of steady flight conditions:

- kinematics equivalence [Approach A]: helicopter steady flight with the same local values of $\mu$ and $\gamma$;

- gravitational and inertial longitudinal loads equivalence [Approach B]: helicopter steady flight with the same local values of $\mu$ and longitudinal 
plane components of weight and inertial loads in a wind axis frame;

- main rotor loads and attitude equivalence [Approach C]: main rotor steady flight corresponding to the same local values of $\mu, F_{z}, M_{x}, M_{y}$ and $\alpha_{s h}$.

A low-fidelity main rotor model suited for flight dynamics is applied in the inverse dynamics tool. However, the prediction of acoustic disturbance generated by BVI phenomena requires accurate evaluation of blade dynamics and aerodynamics. Thus, for each equivalent steady flight considered, highfidelity, aeroelastic, aerodynamic and aeroacoustic main rotor solvers are applied to evaluate the corresponding noise hemisphere. The aeroelastic tool is based on a beam-like rotor blade modelling coupled with three-dimensional, free-wake aerodynamics. As explained in more detail in Appendix B, the aeroelastic response is obtained by application of a Galerkin method for spatial integration, followed by a harmonic balance approach for time integration $[25,29,28]$. The aerodynamic solver is derived from the application of a boundary element method (BEM) formulation for the solution of free-wake, unsteady, potential flows, well suited for analysing configurations where strong BVIs occur $[26,27]$. The aeroacoustic field is derived through the boundary integral solution of the Ffowcs-Williams Hawkings equation widely known as Farassat Formulation 1A [30, 27]. A brief outline of the aerodynamic and aeroacoustic solution approaches applied is provided in Appendix B.

The proposed analysis is focussed on the noise radiated by the main rotor only, in that it is the helicopter component where the strongest BVI phenomena related to approach descent flights take place. Anyway, the investigation process proposed in this work can be applied for the analysis of the acoustic disturbance generated by the main rotor-tail rotor system, or even the complete helicopter configuration.

\subsection{Approach A}

For Approach A, the equivalent noise hemisphere is evaluated as follows: given $\mu$ and $\gamma$, (i) first, considering the same rotorcraft model implemented in the inverse flight dynamics tool, the corresponding steady helicopter trim problem is solved (i.e., constant pilot commands and vehicle attitude fitting the steady rectilinear flight with prescribed $\mu$ and $\gamma$ are determined, assuming zero sideslip angle); then (ii) the high-fidelity, main rotor aeroelastic solver is 
applied to evaluate the isolated main rotor steady operative condition (i.e., with constant blade pitch controls and steady-periodic bending-torsion deflections) corresponding to the same $F_{z}, M_{x}, M_{y}$ and $\alpha_{s h}$ identified by the steady helicopter trim (in the following, this procedure will be referred to as rotor trim); next (iii) blade deformed kinematics and blade loads from the free-wake, unsteady, potential-flow solver are used as input to the aeroacous-

tic formulation predicting noise distribution over a hemisphere surrounding the helicopter.

\subsection{Approach $B$}

In Approach $\mathrm{B}$, for a given advance ratio, $\mu$, first the steady helicopter trim is determined, as associated to a flight condition with vehicle weight components parallel and orthogonal to the trajectory that equal those of the sum of weight and inertial loads evaluated at the specific point along the unsteady flight trajectory. This may be obtained by suitably defining a steady flight climb angle, $\gamma$, and scaling the helicopter weight (in other words, it means equivalence of steady and unsteady flights in terms of wind-axes, longitudinal load factors, if related to a common nominal value of helicopter weight). Note that, it implies equivalent disk loading magnitude and disk orientation with respect to wind axes (namely, rotor thrust and tip-pathplane angle). The next steps of the process implemented to evaluate the noise hemisphere are identical to steps (ii)-(iii) described for Approach A.

\subsection{Approach $C$}

Approach $\mathrm{C}$ does not require the determination of an equivalent helicopter steady flight condition, hence step (i) of Approaches A and B is unnecessary. The starting point (similarly to point (ii) of Approaches A and B) consists in searching the isolated main rotor trim associated to the values of $\mu, \alpha_{s h}$, $F_{z}, M_{x}$ and $M_{y}$ directly evaluated at the given trajectory point during the unsteady maneuver. Then, step (iii) of Approaches A and B is applied. Note that, this approach is not related to a helicopter steady flight, and it can be considered as the noise simulation based on isolated main rotor predictions closest to the fully unsteady solution. The comparison of the results from Approaches $\mathrm{A}$ and $\mathrm{B}$ with those from Approach $\mathrm{C}$ may be exploited to assess the level of noise equivalence of the helicopter steady flights defined in Approaches A and B with the unsteady maneuver flight (indeed, rotor blade dynamics is typically much faster than helicopter dynamics, and hence rotor steady aeroelastic/aeroacoustic solutions matching instantaneous values 
of hub loads, advance ratio and shaft attitude, yield reliable simulations of acoustic disturbance emitted during an unsteady maneuver).

\section{Numerical Investigation}

The numerical investigation on the assessment of noise prediction techniques described in Section 2 concerns the unsteady flight of a lightweight helicopter inspired by the BO105. The BO105 is a relatively small, multipurpose helicopter built by formerly MBB (now Eurocopter), with an empty mass of about $1200 \mathrm{~kg}$ and a maximum gross mass of $2300 \mathrm{~kg}$. Typical uses of the highly maneuverable BO105 helicopter are transport, offshore, police, and military missions. The BO105 has a four-bladed, hingeless main rotor of $4.91 \mathrm{~m}$ radius, with blade precone angle of $2.5^{\circ}$; the rotor shaft is tilted forward by $3^{\circ}$. The two-bladed teetering tail rotor operates in pushing configuration; the tail surfaces are composed of a horizontal stabilizer and a vertical empennage, both fixed to the fuselage (a sketch of the helicopter with the relevant geometrical data is shown in Fig. 2).

For the numerical simulations presented in this work, the fuselage is assumed to be rigid; in the flight dynamics solver, the main rotor is described through a simplified model composed of rigid-body, hinged blades with a torsion spring taking into account control chain dynamics effects, while more realistic hingeless, elastic rotor blades have been considered in aeroelastic and aerodynamic tools. The main geometrical, inertial and elastic characteristics of the helicopter model used here are related to those provided in Ref. [31], and have already been applied by some of the authors for rotorcraft-pilot coupling investigations (see, for instance, Ref. [32]).

\subsection{Trajectory Definition}

The test case mission segment considered in the numerical investigation consists of an approach path to a heliport starting from a level, steady rectilinear flight and ending with a lower altitude, lower airspeed, differently oriented, rectilinear, flight condition. Specifically, in order to consider effects from different unsteady maneuvers, the trajectory is defined by means of a sequence of the following flight segments

- straight, uniform, level flight

- straight, decelerating, descent flight 
- steady, banked, level turn

- straight, uniform, level flight at very low speed

for a total time duration of $38 \mathrm{~s}$, that corresponds to the evolutions of center of mass velocity, $V$, flight path climb angle, $\gamma$, and azimuthal angle, $\chi$, presented in Fig. 3. The resulting helicopter center of mass trajectory represented in a ground-fixed frame of reference is depicted in Fig. 4.

\subsection{Inverse Flight Dynamics Simulation}

The inverse flight dynamics simulation for the helicopter trajectory defined above is carried out imposing zero sideslip angle along the entire flight path. The resulting main rotor pilot control commands are depicted in Fig. 5, whereas Fig. 6 presents the time histories of the helicopter attitude angles. As described in Section 2, the time histories of loads generated by the main rotor and transmitted to the fuselage through the hub are among the most important elements for properly characterizing noise hemispheres. The loads determined for the trajectory here considered are presented in Figs. 7 (forces) and 8 (moments). It is worth noting that, during the decelerated descent flight a remarkable pitch-up helicopter attitude makes the main rotor operate in almost autorotation condition, as demonstrated by the very small value of torque required to drive the shaft (see $M_{z}$ time-history in Fig. 8). Loads in Figs. 7 and 8 are the reference values used to trim the equivalent steady flight of the isolated elastic main rotor for Approach C (see Section 2).

\subsection{Noise Hemispheres Prediction and Correlation}

Along the defined trajectory, the four points numbered sequentially in Fig. 4 (indicated by square marks in Figs. 3 and 5-8) are selected for noise hemisphere analyses. They correspond to specific characteristic flight conditions: point 1 represents a steady, straight, level flight, point 2 is a point of the transition maneuver from level to descent flight, point 3 is the initial point of deceleration, whereas point 4 is the mid point of the turning maneuver.

As described in Section 2, for each point of analysis defined along the unsteady flight path, the isolated elastic main rotor is trimmed as described in Approaches A, B and C, and the corresponding aeroelastic/aerodynamic response is used as input to the aeroacoustic solver. The aeroelastic response 
analyses have been performed considering 3 shape functions for each flap, lead-lag and torsion deformations, with the Lagrangian coordinates described by 5 harmonics. Furthermore, the aerodynamic BEM solver has been applied considering a blade surface discretization with 20 upper and lower chordwise panels, 24 spanwise panels, with the time interval in the time-marching, freewake solution corresponding to a blade azimuthal step of $2 \pi / 216 \mathrm{rad}$.

The noise hemisphere is evaluated at a distance of $150 \mathrm{~m}$ from the main rotor hub, in terms of distribution of the overall sound pressure level (OASPL) associated to the acoustic disturbance emitted by the main rotor. The hemisphere is fixed to the vehicle frame of reference, with the equatorial plane parallel to the cabin floor. As a benchmark for the noise predicted along the unsteady flight considered, Figure 9 shows the noise hemisphere at point 1 represented through a planar view, as obtained from projection onto the equatorial plane (the same view is used to depict all the noise hemispheres presented in what follows). At this trajectory point, where the helicopter is in level, rectilinear, steady flight, inertial loads are absent and predictions from Approaches A, B and C coincide.

Figure 10 presents the noise hemispheres at trajectory point 2 evaluated through the three approaches introduced. In this case, Approach A considerably overestimates the acoustic disturbance emitted by the main rotor, whereas Approach B yields a noise hemisphere well correlated with that from Approach C, both in terms of noise intensity and directivity. Indeed, at point 2 the curvature of the trajectory generates the onset of inertial loads, that in turn induce a significant reduction of the disk loading with respect to the flight condition at point 1 (Fig. 7 shows that the hub forces at point 2 are more than $20 \%$ smaller than those at point 1 ). Hence, in this case, the characterization of the noise hemisphere through mere kinematic equivalence (Approach A) yields a poor correlation between the unsteady flight condition and the corresponding steady-state approximation. This is confirmed by the differences reported in Table 1 among pilot controls and shaft angle identified by the main rotor trim in Approach A and those given by Approaches B and $\mathrm{C}$, as well as by the corresponding tip flap deflections shown in Fig. 11. The greater disk loading predicted by Approach A is also associated to stronger wake vortices and a less regular wake surface predicted by the aerodynamic BEM tool (as illustrated in Fig. 12, where it is compared with wake surfaces given by Approach B and C). In turn, this strongly affects miss distance and BVI events simulation.

At trajectory point 3 , inertial loads due to acceleration arise, causing 
Table 1

Pitch controls and shaft angle at point 2

\begin{tabular}{lcccc}
\hline & $\theta_{0}[\mathrm{deg}]$ & $A_{1 s}[\mathrm{deg}]$ & $B_{1 s}[\mathrm{deg}]$ & $\alpha_{s h}[\mathrm{deg}]$ \\
\hline Approach A & 3.7 & -1.5 & 0.9 & -7.2 \\
Approach B & 3.3 & -1.4 & 1.0 & -9.0 \\
Approach C & 3.2 & -1.3 & 1.1 & -8.8 \\
\hline
\end{tabular}

remarkable variations of hub loads $F_{x}$ and $M_{x}$ with respect to their values in rectilinear, steady, level flight (see Figs. 7 and 8). Again, as demonstrated in Fig. 13, their presence makes the noise hemisphere from Approach $\mathrm{A}$ less correlated to that from Approach $\mathrm{C}$, with respect to the one determined through Approach B. However, it is worth noting that inertial loads generated at point 3 are such that Approach A yields better predictions as compared to those given at point 2, where a higher load factor increment is associated to the maneuver.

Finally, the noise hemisphere at the turning point 4 is examined. At this point, the inertial loads related to the coordinated turn correspond to a load factor of 1.1, as it may be inferred from the roll angle history in Fig. 6. The presence of inertial loads induces increase of disk loading with respect to that required for the steady, straight level flight considered in Approach A, and this explains the significantly lower noise level predicted by this approach as compared to those from Approaches B and C shown in Fig. 14. Note that, a certain degree of inaccuracy in predicting noise directivity is observed in the results from Approach B.

From the results presented for the trajectory points examined, some considerations regarding acoustic equivalence among different helicopter flight conditions may be drawn. Indeed, helicopter rotor aerodynamics and noise emission are strictly correlated to the components of the hub loads transmitted to the fuselage or, equivalently, to disk loading and orientation with respect to the relative wind. This is particularly true for approach flights, where the miss distance between blades and wake vortices plays a crucial role in the onset of BVI phenomena. In rectilinear, steady flight, rotor disk loading and orientation is univocally associated to advance ratio and flight path angle through the rotorcraft trim conditions. However, in maneuvering flight, inertial loads arising affect rotor thrust and aeroelastic response, and hence tip-path-plane angle. This implies that, in this case, local values along 
the trajectory flown of advance ratio and flight path angle are not sufficient to identify rotor disk loading and orientation with respect to the relative wind and, as a consequence, emitted noise. Thus, three parameters are needed to characterize noise hemispheres associated to the acoustic emissions of helicopters in maneuvering flights, with explicit inclusion, in addition to the advance ratio, of rotor thrust coefficient and tip-path-plane angle.

\section{Conclusions}

Different approaches based on steady flight aeroelastic, aerodynamic and aeroacoustic predictions have been examined for assessing their capability to simulate the acoustic impact of unsteady approach maneuvers of helicopters, where BVI phenomena play a crucial role. The purpose of the analysis has been the identification of those flight parameters suited for the characterization of the steady-flight noise hemispheres that form databases typically applied in tools for rotorcraft ground acoustic impact evaluation, or optimal search of minimum-noise helicopter trajectories. This has been accomplished by selecting a limited number of steady flight parameters, whose close similarity with those instantaneously occurring during an unsteady flight guarantees the acoustic equivalence in terms of radiated noise hemispheres. The outcomes of the analyses have demonstrated that the local similarity of only two kinematic parameters (namely, advance ratio and flight path angle) does not assure the local acoustic equivalence of steady and unsteady flights. Rather, it has been shown that a satisfactory level of acoustic equivalence is reached when the steady flight presents, in addition to the advance ratio, a system of rotorcraft loads close to that of the maneuvering flight, where an important role may be played by the inertial terms. No matter the way the load equivalence is accomplished (weight increase, tailored flight path angle...), the important issue is that the rotor disk load components with respect to the trajectory are similar. Indeed, acoustic phenomena dominated by BVI noise are strongly dependent on main rotor wake vortices strength (strictly related to rotor thrust) and their miss distance from blades (affected by rotor disk orientation with respect to the relative wind). Therefore, at least three parameters including disk loading, are needed to adequately characterize noise hemispheres: advance ratio and disk load components along longitudinal wind axes, or advance ratio, rotor thrust and tip-path-plane angle, for instance. Conversely, considering only kinematic similarity may yield steady flight acoustic predictions completely uncorrelated with those obtained from 
the unsteady maneuver. Furthermore, it has been proven that, as expected, the acoustic equivalence also implies (or better, is the result of) close correlation of aerodynamic and aeroelastic responses.

\section{Appendix A. Inverse Simulation of Helicopter Motion}

An inverse simulation (IS) algorithm allows the determination of the control law that realizes a prescribed trajectory for fixed- and rotary-wing aircraft [21]. A model predictive control (MPC) scheme [23] is used for solving the IS problem. This scheme addresses issues related to the presence of transmission zeros and non-minimum phase response, as well as of violations of physical limits in the system as, for instance, constraints on control travel and control rate or limitations of rotor torque. Unlike the formulation proposed in [23], where a simplified, lower-order model of the helicopter is used to derive the inverse solution, the adopted approach manages the same complexity of the rotorcraft model in both phases of control determination and solution propagation through, respectively, inverse and direct simulation. For the present application, the guidance term of the MPC algorithm allows one to asymptotically recover the desired trim equilibria during steady state flight phases.

The inverse simulation step is based on the so-called integration method $[21,22]$. The values of four control variables are determined at each time step during the maneuver by solving a local optimization problem, that consists in enforcing a terminal constraint on three output variables (namely, the velocity components in the Earth-fixed reference frame), that are required to achieve a prescribed desired value, as specified in the different phases

of the maneuver. A further constraint is required to match the number of controls, expressed as either zero sideslip angle or zero yaw rate, the latter being enforced at the end of the landing maneuver, when a straight flight path at very low flight speed needs to be realized.

The rotorcraft is assumed as rigid and its motion is described in terms of center of mass displacement and yaw, pitch and roll attitude angles. The main rotor model is based on a second-order dynamics for flap and lag angles ( $\beta$ and $\zeta$, respectively), assuming rigid and articulated blades. An equivalent elastic hinge model is used to take into consideration the effect of blade flexural stiffness for the hingeless rotor of the BO105. As for torsional deflections, the dynamic twist of the blade is expressed as the product of a generalized variable $(\phi)$, featuring a second-order dynamics times a shape function. The 
aerodynamic loads on 2-D blade elements are determined as a function of local angle of attack and Mach number, taking into account compressibility effects and the stall of the retreating blade. Loads are numerically integrated over the blade span. Inflow dynamics of the main rotor is represented through a three-state, first-order model. Tail rotor thrust is evaluated by means of momentum theory with a first-order dynamics for the uniform inflow. Fuselage aerodynamic model is given in tabular form in terms of force and moment coefficient depending on angle of attack and sideslip angle. A detailed description of the methodology adopted for rotorcraft modelling and solution algorithm for the inverse simulation problem can be found in Ref. $[24]$.

As a result of the modelling approach adopted, the state vector is given by $\mathbf{x}^{T}=\left(\mathbf{x}_{\text {fus }}^{T}, \mathbf{x}_{R}^{T}, \mathbf{x}_{i n}^{T}\right)$, where the elements of $\mathbf{x}_{\text {fus }}^{T}=(u, v, w ; p, q, r ; \phi, \theta, \psi ; x, y, h)$ are rigid-body states, that is, linear and angular velocity components, Euler angles and c.g. coordinates, and $\mathbf{x}_{R}^{T}=\left(\mathbf{x}_{b l_{1}}^{T}, \ldots, \mathbf{x}_{b l_{i}}^{T}, \ldots, \mathbf{x}_{b l_{N}}^{T}\right)$ represents rotor states in terms of individual blade flap and lag degrees of freedom, being $\mathbf{x}_{b l_{i}}^{T}=\left(\beta_{i}, \dot{\beta}_{i}, \zeta_{i}, \dot{\zeta}_{i}, \phi_{i}, \dot{\phi}_{i}\right)$. Finally, inflow states are collected in the vector $\mathbf{x}_{i n}^{T}=\left(\nu_{0}, \nu_{c}, \nu_{s}, \nu_{T R_{0}}\right)$, where the first three elements allow for the description of main rotor inflow in the form $w_{i}=\left[\nu_{0}+r\left(\nu_{c} \cos \psi+\nu_{s} \sin \psi\right)\right] \Omega R$. whereas the last one provides tail rotor uniform nondimensional inflow velocity.

The model is written in concise form as $\dot{\mathbf{x}}=\mathbf{f}(t ; \mathbf{x}, \mathbf{u}) ; \mathbf{y}=\mathbf{g}(\mathbf{x})$, where $\mathbf{x} \in \mathcal{R}^{40}$ is the state vector, $\mathbf{y} \in \mathcal{R}^{4}$ is the vector of tracked outputs and the control vector $\mathbf{u}=\left(\theta_{0}, A_{1 s}, B_{1 s} ; \theta_{0_{T R}}\right)^{T}$ features main and tail rotor collective pitch controls $\left(\theta_{0}, \theta_{0_{T R}}\right)$ and lateral and longitudinal cyclic pitch commands $\left(A_{1 s}, B_{1 s}\right)$.

Once the output trajectory $\mathbf{y}_{\text {des }}(t)$ is specified over the maneuver time interval, the inverse problem is solved starting from the initial condition $\mathbf{x}_{k}=$ $\mathbf{x}\left(t_{k}\right)$ at the end of the previous time-step. The procedure is based on three steps, that is: i) determination of the increment $\Delta \mathbf{y}_{k}^{*}$ for the output variables, being $\Delta \mathbf{y}_{k}^{*}=\Delta \mathbf{y}_{\text {des }}+\Delta \mathbf{y}_{\text {guid }}$, where $\Delta \mathbf{y}_{\text {des }}=\mathbf{y}_{\text {des }}\left(t_{k}+T\right)-\mathbf{y}_{\text {des }}\left(t_{k}\right)$ and $\Delta \mathbf{y}_{\text {guid }}=K\left[\mathbf{y}_{\text {des }}\left(t_{k}\right)-\mathbf{g}\left(x_{k}\right)\right]$, ii) evaluation of the command value $\mathbf{u}_{k}^{*}$ that achieves the desired variation of the output, where the receding time-horizon $T=N \Delta t$ allows for an adequate settling time for the uncontrolled dynamics; and iii) forward simulation to propagate the state of the model from time $t_{k}$ through $t_{k+1}=t_{k}+\Delta t$ for the constant control input $\mathbf{u}_{k}^{*}$ determined by means of the inverse solution at step ii). 


\section{Appendix B. Aeroelastic, Aerodynamic and Aeroacoustic Main Ro- tor Modelling}

The simulation of the acoustic disturbance generated by rotors in BVI conditions is a multidisciplinary task: blade aeroelasticity and aerodynamics accurate modelling are required to yield the blade surface pressure distribution that, in turn, is the input to an aeroacoustic tool providing the radiated noise. Indeed, blade-wake miss distance plays a crucial role, and hence the evaluation of blade deformation and wake shape is essential. The following sections provide a brief outline of the methodologies applied in this work to determine noise hemispheres.

\section{Appendix B.1. Rotor Aeroelastic Modelling}

Aeroelastic responses are obtained by combining a blade structural dynamics model with a three-dimensional, free-wake, aerodynamic formulation.

Blade structural dynamics is described through a beam-like model. It derives from a nonlinear, bending-torsion formulation valid for slender, homogeneous, isotropic, nonuniform, twisted blades, undergoing moderate displacements [33]. The radial displacement is eliminated from the set of equations by solving it in terms of local tension, and thus the resulting structural operator consists of a set of coupled nonlinear differential equations governing the bending of the elastic axis and the blade torsion [34].

The evaluation of the aerodynamic loads is obtained by a boundary element method for the solution of a boundary integral equation approach, suited for the analysis of potential flows around helicopter rotors in arbitrary flight condition [26], briefly outlined in the next section.

Coupling blade structural dynamics with aerodynamic loads yields an aeroelastic integro-partial differential system of equations. These are spatially integrated through the Galerkin approach, with the description of elastic axis deformation and cross-section torsion as linear combinations of shape functions satisfying homogeneous boundary conditions. This yields a set of nonlinear, ordinary differential equations of the type

$$
\mathbf{M}(t) \ddot{\mathbf{q}}+\mathbf{C}(t) \dot{\mathbf{q}}+\mathbf{K}(t) \mathbf{q}=\mathbf{f}_{s t r}^{n l}(t, \mathbf{q})+\mathbf{f}_{a e r}(t, \mathbf{q})
$$

where $\mathbf{q}$ denotes the vector of the Lagrangian coordinates, $\mathbf{M}, \mathbf{C}$, and $\mathbf{K}$ are time-periodic, mass, damping, and stiffness structural matrices representing the linear structural terms. Non-linear structural contributions are collected in the forcing vector $\mathbf{f}_{s t r}^{n l}(t, \mathbf{q})$, whereas vector $\mathbf{f}_{a e r}(t, \mathbf{q})$ collects the generalized 
aerodynamic forces. For the prediction of the aeroelastic periodic response during steady flight, the aeroelastic system in Eq. (B.1) is solved by using the harmonic balance approach $[25,28]$. It is a methodology suitable for the analysis of the asymptotic solution (as time goes to infinity) of differential equations forced by periodic terms. Because of the presence of nonlinear contributions deriving both from structural terms and from the free-wake aerodynamic loads prediction, the final system has to be solved using an iterative approach. To this aim, the Newton-Raphson procedure is applied.

\section{Appendix B.2. Rotor Aerodynamic Solver}

Considering a potential velocity field, $\mathbf{v}=\nabla \varphi$, the rotor aerodynamics formulation applied assumes the potential field, $\varphi$, to be given by the superposition of an incident field, $\varphi_{I}$, and a scattered field, $\varphi_{S}$ (i.e., $\varphi=\varphi_{I}+\varphi_{S}$ ). The scattered potential is generated by sources and doublets over the surfaces of the blades, $S_{B}$, and by doublets over the wake portion that is very close to the trailing edge from which emanated (near wake, $S_{W}^{N}$ ). The incident potential is due to doublets distributed over the complementary wake region that compose the far wake $S_{W}^{F}[26]$. The wake surface partition is such that the far wake is the only wake portion that may come in contact with blades and generate BVI effects. The incident potential is discontinuous across $S_{W}^{F}$, whereas the scattered potential is discontinuous across $S_{W}^{N}$ and is represented by $[26]$

$$
\varphi_{S}(\mathbf{x}, t)=\int_{S_{B}}\left[G\left(v_{n}-u_{n}\right)-\varphi_{S} \frac{\partial G}{\partial n}\right] \mathrm{d} S(\mathbf{y})-\int_{S_{W}^{N}} \Delta \varphi_{S} \frac{\partial G}{\partial n} \mathrm{~d} S(\mathbf{y})
$$

where $G=-1 / 4 \pi r$ is the unit-source solution of the three-dimensional Laplace equation, with $r=\|\mathbf{y}-\mathbf{x}\|$, while $\Delta \varphi_{S}$ is the potential jump across the wake surface, known from past history of potential discontinuity at the blade trailing edge through the Kutta-Joukowski condition $[35,36]$. In addition, $v_{n}=\mathbf{v}_{B} \cdot \mathbf{n}$, with $\mathbf{v}_{B}$ representing the blade velocity and $\mathbf{n}$ its outward unit normal, whereas $u_{n}=\mathbf{u}_{I} \cdot \mathbf{n}$, with $\mathbf{u}_{I}$ denoting the velocity induced by the far wake.

Considering the far wake discretized into $M$ panels, assuming the potential jump constant over each panel, and recalling the equivalence between surface distribution of doublets and vortices, the incident velocity field is evaluated through the Biot-Savart law applied to the vortices having the shape of the panel contours. In order to assure a regular distribution of the induced 
velocity within the vortex core, and thus a stable and regular solution even in blade-vortex impact conditions, a Rankine finite-thickness vortex model is introduced in the Biot-Savart law [26]. Wake-induced velocity field is applied to evaluate the term $u_{n}$ in Eq. (B.2), as well as the velocity field from which the wake shape evolution is determined in a free-wake analysis. Note that, for an accurate prediction of BVI phenomena, the accurate evaluation of the wake distorted shape is essential in that a crucial role is played by the relative positions between body and wake.

In this formulation, the incident potential affects the scattered potential through the induced-velocity, while the scattered potential affects the incident potential by its trailing-edge discontinuity that is convected along the wake and yields the intensity of the vortices of the far wake [26]. Once the potential field is known, the Bernoulli theorem yields the pressure distribution to be provided to the aeroelastic and aeroacoustic solvers [28].

\section{Appendix B.3. Rotor Noise Radiation}

Noise radiated by rotor blades is evaluated through solution of the wellknown Ffowcs Williams and Hawkings (FW-H) equation [37], which governs the propagation of acoustic disturbances generated aerodynamically by moving bodies.

The boundary integral formulation developed by Farassat known as Formulation 1A [30] is a widely-used and computationally efficient way to determine the acoustic field as solution of the FW-H equation, and is particularly suited for the problems examined here. When the velocity of the rotor blades is far from the transonic/supersonic range, it yields the aeroacoustic field as a superposition of a term, $p_{T}^{\prime}$, depending on blade geometry and kinematics (thickness noise), and of a term, $p_{L}^{\prime}$, that is related to the blade airloads (loading noise). These two noise contributions are given by the following integrals evaluated over the actual blade surface, $S_{B}[30]$

$$
\begin{aligned}
4 \pi p_{T}^{\prime}(\mathbf{x}, t) & =\int_{S_{B}}\left[\frac{\rho_{0} \dot{v}_{n}}{r\left|1-M_{r}\right|^{2}}\right]_{\tau} \mathrm{d} S(\mathbf{y}) \\
& +\int_{S_{B}}\left[\frac{\rho_{0} v_{n}\left(r \dot{\mathbf{M}} \cdot \hat{\mathbf{r}}+c_{0} M_{r}-c_{0} M^{2}\right)}{r^{2}\left|1-M_{r}\right|^{3}}\right]_{\tau} \mathrm{d} S(\mathbf{y})
\end{aligned}
$$




$$
\begin{aligned}
4 \pi p_{L}^{\prime}(\mathbf{x}, t) & =\frac{1}{c_{0}} \int_{S_{B}}\left[\frac{\dot{\tilde{p}} \mathbf{n} \cdot \hat{\mathbf{r}}+\tilde{p} \dot{\mathbf{n}} \cdot \hat{\mathbf{r}}}{r\left|1-M_{r}\right|^{2}}\right]_{\tau} \mathrm{d} S(\mathbf{y}) \\
& +\int_{S_{B}}\left[\frac{\tilde{p} \mathbf{n} \cdot \hat{\mathbf{r}}-\tilde{p} \mathbf{M} \cdot \mathbf{n}}{r^{2}\left|1-M_{r}\right|^{2}}\right]_{\tau} \mathrm{d} S(\mathbf{y}) \\
& +\frac{1}{c_{0}} \int_{S_{B}}\left[\frac{\tilde{p} \mathbf{n} \cdot \hat{\mathbf{r}}}{r^{2}\left|1-M_{r}\right|^{3}}\left(r \dot{\mathbf{M}} \cdot \hat{\mathbf{r}}+c_{0} M_{r}-c_{0} M^{2}\right)\right]_{\tau} \mathrm{d} S(\mathbf{y})(B .
\end{aligned}
$$

where $c_{0}$ and $\rho_{0}$ are, respectively, the speed of sound and the density in the undisturbed medium, whereas $\tilde{p}=\left(p-p_{0}\right)$ with $p_{0}$ representing the undisturbed medium pressure, $\mathbf{M}=\mathbf{v}_{B} / c_{0}, M=\|\mathbf{M}\|$, and $M_{r}=\mathbf{M} \cdot \hat{\mathbf{r}}$ with $\hat{\mathbf{r}}=\mathbf{r} /\|\mathbf{r}\|$. In addition, $\dot{\mathbf{n}}$ and $\dot{\mathbf{M}}$ denote time derivatives, respectively, of the outward blade surface unit normal vector and of the local blade velocity Mach vector, as observed in a frame of reference fixed with the undisturbed medium, whereas the notation $[\ldots]_{\tau}$ indicates that these quantities must be evaluated at the emission time, $\tau$, i.e., the time at which the signal arriving in $\mathbf{x}$ at time $t$ started from $\mathbf{y} \in S_{B}[30]$.

In problems dealing with weakly loaded rotors, thickness and loading noise are comparable. However, when strongly loaded rotors are examined, the thickness noise contribution tends to be negligible and the acoustic disturbance is dominated by the loading noise. Rotors in BVI conditions fall within this category of acoustic phenomena.

\section{Acknowledgments}

This work has been partially supported by the Project MANOEUVRES, financed under the Clean Sky Joint Undertaking Programme (Grant agreement N. 620068).

\section{References}

[1] Y.H. Yu, B. Gmelin, W. Splettstoesser, J.J. Philippe, P. Prieur, T.F. Brooks, Reduction of helicopter blade-vortex interaction noise by active rotor control technology, Progress in Aerospace Sciences, 33 (9-10) (1997) 647-687.

[2] P.C. Chen, J.D. Baeder, R.A.D. Evans, J. Niemczuk, Blade-vortex interaction noise reduction with active twist smart rotor technology, Smart Materials and Structures, 10 (1) (2001) 77-85. 
[3] D. Patt, L. Liu, P.P. Friedmann, Simultaneous vibration and noise reduction in rotorcraft using aeroelastic simulation, Journal of the American Helicopter Society, 51 (2) (2006) 127-140.

[4] R.D. JanakiRam, B.W. Sim, C. Kitaplioglu, F.K. Straub, Blade-vortex interaction noise characteristics of a full-scale active flap rotor, Proceedings of the 65th Annual Forum of the American Helicopter Society, Grapevine, Texas, 2009.

[5] R. Muller, The influence of winglets on rotor aerodynamics, Proceedings of the 12nd European Rotorcraft Forum, Garmisch, Germany, 1986.

[6] A. Desopper, P. Lafon, P. Prieur, Effect of an anhedral sweptback tip on the performance of a helicopter rotor, Proceedings of the 13rd European Rotorcraft Forum, Arles, France, 1987.

[7] D. Favier, C. Maresca, E. Berton, P. Plantin de Hugues, Investigation of the tips shape influence on the flowfield around hovering rotor blades, Proceedings of the 22nd AIAA Fluid Dynamics Conference, Honolulu, Hawaii, 1991.

[8] P. Rauch, M. Gervais, P. Cranga, A. Baud, J.F. Hirsch, A. Walter, P. Beaumier, Blue edge: the design, development and testing of a new blade concept, Proceedings of the 67th Annual Forum of the American Helicopter Society, Virginia Beach, VA, 2011.

[9] Y.H. Yu, Rotor blade-vortex interaction noise, Progress in Aerospace Sciences, 36 (2) (2000) 97-115.

[10] D.A. Conner, J.A. Page, A tool for low noise procedures design and community noise impact assessment: the rotorcraft noise model (RNM), Proceedings of Heli Japan 2002, Tochigi, Japan, 2002.

[11] D.A. Conner, C.L. Burley, C.D. Smith, Flight acoustic testing and data acquisition for the rotor noise model (RNM). Proceedings of the 62nd Annual Forum of the American Helicopter Society, Phoenix, Arizona, 2006 .

[12] J. Yin, H. Buchholz, Toward noise abatement flight procedure design: DLR rotorcraft noise ground footprints model, Journal of the American Helicopter Society 52 (2) (2007) 90-98. 
[13] A. Le Duc, P. Spiegel, F. Guntzer, M. Kummer, J. Götz, Modelling of helicopter noise in arbitrary maneuver flight using aeroacoustic database, Proceedings of the 9th Onera-DLR Aerospace Symposium (ODAS 2008), Chtillon, France, 2008.

[14] T. Tsuchiya, H. Ishii, J. Uchida, H. Ikaida, H. Gomi, N. Matayoshi, Y. Okuno, Flight trajectory optimization to minimize ground noise in helicopter landing approach, Journal of Guidance, Control and Dynamics 32 (2) (2009) 605-615.

[15] H.G. Visser, M.D. Pavel, S.F. Tang, Optimization of rotorcraft simultaneous non-interfering noise abatement approach procedures, Proceedings of the 47th AIAA Aerospace Sciences Meeting, Orlando, FL, USA, 2009.

[16] S. Hartjes, Y. Buys, H.G. Visser, M.D. Pavel, M. Gennaretti, G. Bernardini, M. Arntzen, Optimization of rotorcraft noise abatement trajectories, Proceedings of the Internoise 2012/ASME NCAD Meeting, New York City, NY, Aug 2012.

[17] R.A. Morris, K.B. Venable, J. Lindsey, Automated design of noiseminimal, safe rotorcraft trajectories, Proceedings of the 68th Annual Forum of the American Helicopter Society, Fort Worth, Texas, 2012.

[18] Office of Environment and Energy, Integrated Noise Model (INM) Version 5.1 Technical Manual, Rept. FAA-AEE-97-04, Federal Aviation Agency, 1997.

[19] J.P. Clarke, R.J. Hansman, Systems analysis of noise abatement procedures enables by advanced flight guidance technology, AIAA Paper 97-0490, Proceeding of the 35th AIAA, Aerospace Sciences Meeting \& Exhibit, Reno, NV, 1997.

[20] H.G. Visser, R.A.A. Wijnen, Optimization of noise abatement departure trajectories, Journal of Aircraft 38 (4) (2001) 620-627.

[21] R.A. Hess, C. Gao, A generalized algorithm for inverse simulation applied to helicopter maneuvering flight, Journal of the American Helicopter Society 16 (5) (1993) 3-15. 
[22] G. Avanzini, G. De Matteis, Two-timescale inverse simulation of a helicopter model, Journal of Guidance, Control, and Dynamics 24 (2) (2001) 330-339.

[23] G. Avanzini, D. Thomson, A. Torasso, Model predictive control architecture for rotorcraft inverse simulation, Journal of Guidance, Control, and Dynamics 36 (1) (2013) 207-217.

[24] A. Torasso, Low-order models and numerical techniques for the analysis of rotorcraft flight mechanics, PhD Thesis, Politecnico di Torino, 2012.

[25] M. Gennaretti, G. Bernardini, Aeroelastic response of helicopter rotors using a 3-D unsteady aerodynamic solver, The Aeronautical Journal 110 (1114) (2006) 793-801.

[26] M. Gennaretti, G. Bernardini, Novel boundary integral formulation for blade-vortex interaction aerodynamics of helicopter rotors, AIAA Journal 45 (6) (2007) 1169-1176.

[27] G. Bernardini, J. Serafini, S. Ianniello, M. Gennaretti, Assessment of computational models for the effect of aeroelasticity on BVI noise prediction, International Journal of Aeroacoustics 6 (3) (2007) 199-222.

[28] G. Bernardini, J. Serafini, M. Molica Colella, M. Gennaretti, Analysis of a structural-aerodynamic fully-coupled formulation for aeroelastic response of rotorcraft, Aerospace Science and Technology 29 (1) (2013) 175-184.

[29] P. Masarati, G. Quaranta, M. Gennaretti, J. Serafini, Aeroservoelastic analysis of rotorcraft-pilot coupling: a parametric study, Proceedings of the 66th Annual Forum of the American Helicopter Society, Phoenix, AZ, May 2010.

[30] F. Farassat, Derivation of formulations 1 and 1A of Farassat, NASA TM 214853, 2007.

[31] B. Dang-Vu, P. Masarati, G. Quaranta, M. Gennaretti, M. Jump, I. Fuiorea, A. Ionita, Generic helicopter database, Technical Report Deliverable No. D3.1, EU funded project ARISTOTEL (GA no. 266073), 2011. 
[32] M. Gennaretti, J. Serafini, P. Masarati, G. Quaranta, Effects of biodynamic feedthrough in rotorcraft/pilot coupling: collective bounce case, Journal of Guidance, Control, and Dynamics 36 (6) (2013) 1709-1721.

[33] D.H. Hodges, E.H. Dowell, Nonlinear equation for the elastic bending and torsion of twisted nonuniform rotor blades, NASA TN D-7818, 1974.

[34] D.H. Hodges, R.A. Ormiston, Stability of elastic bending and torsion of uniform cantilever rotor blades in hover with variable structural coupling, NASA TN D-8192, 1976.

[35] M. Gennaretti, L. Luceri, L. Morino, A unified boundary integral methodology for aerodynamics and aeroacoustics of rotors, Journal of Sound and Vibration 200 (4) (1997) 467-489.

[36] L. Morino, G. Bernardini, Singularities in BIE's for the Laplace equation; Joukowski trailing-edge conjecture revisited, Journal of Engineering Analysis with Boundary Elements, 25 (2001) 805-818.

[37] J.E. Ffowcs Williams, D.L. Hawkings, Sound generation by turbulence and surfaces in arbitrary motion, Philosophical Transactions of the Royal Society of London 264 (1151) (1969) 321-342. 


\section{Figures}

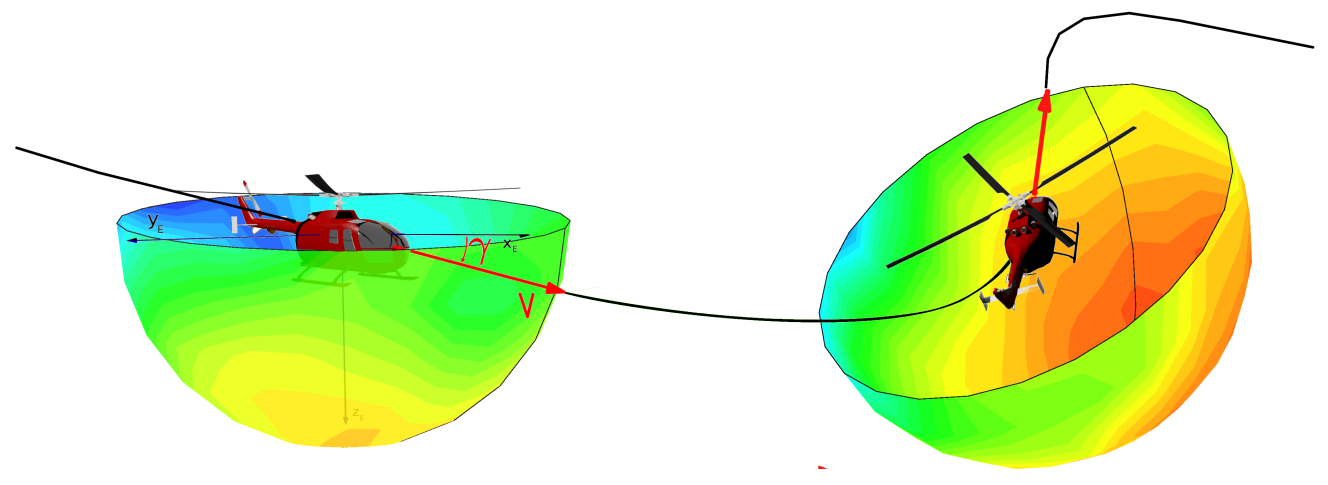

Figure 1: Noise hemispheres concept for optimal trajectory search.

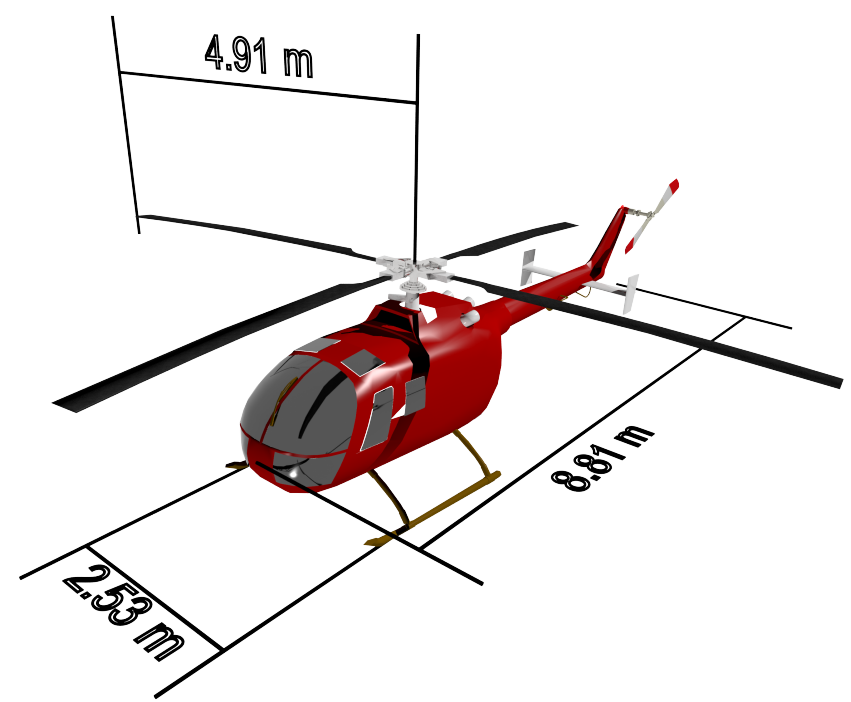

Figure 2: Sketch of helicopter BO105. 

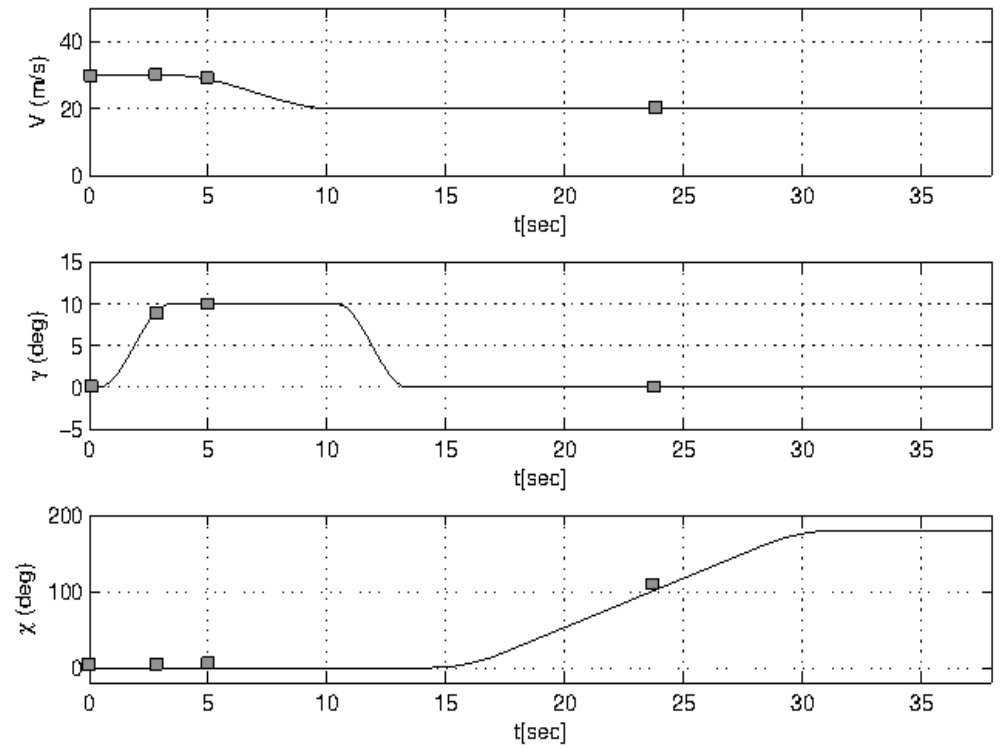

Figure 3: Time-histories of velocity and trajectory climb and azimuthal angles.

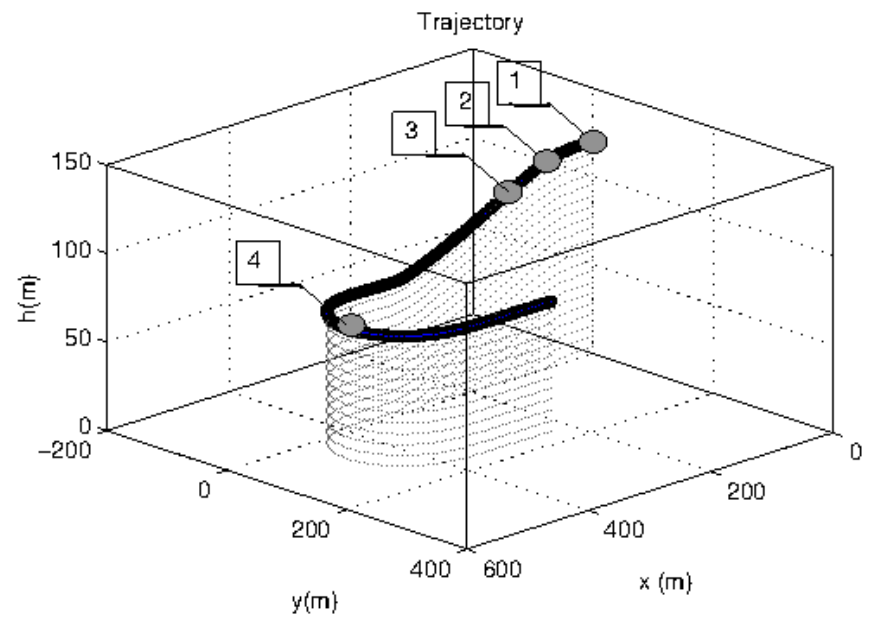

Figure 4: Helicopter trajectory. 

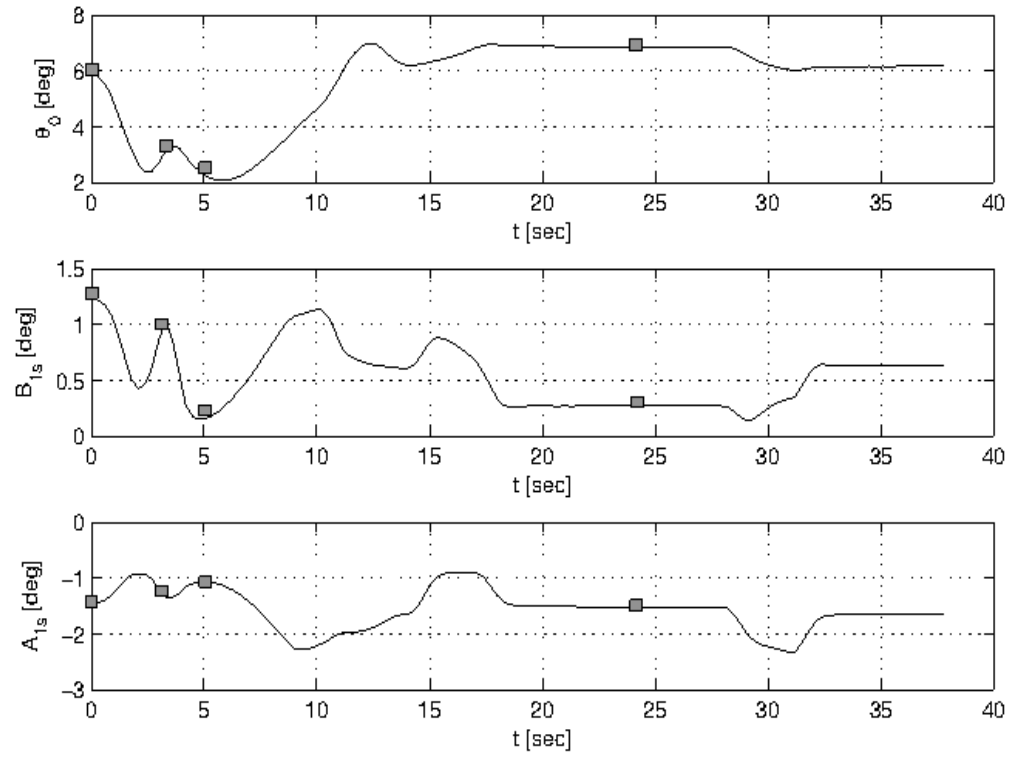

Figure 5: Time-histories of rotor blade collective and cyclic controls.
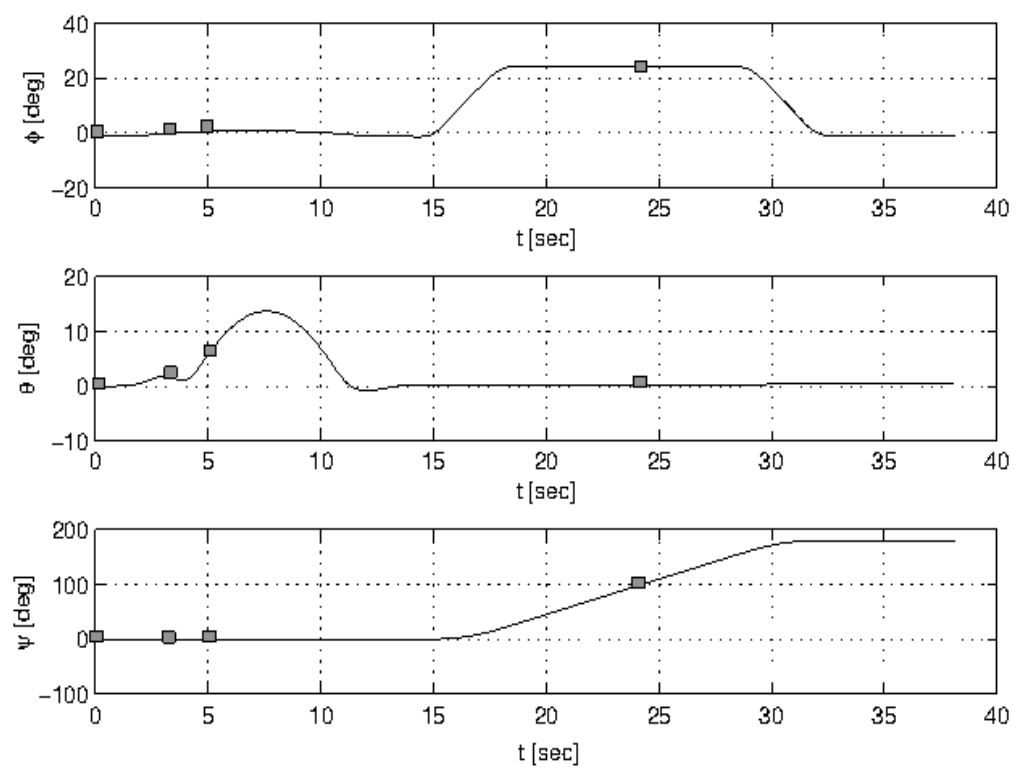

Figure 6: Time-histories of helicopter attitude angles. 

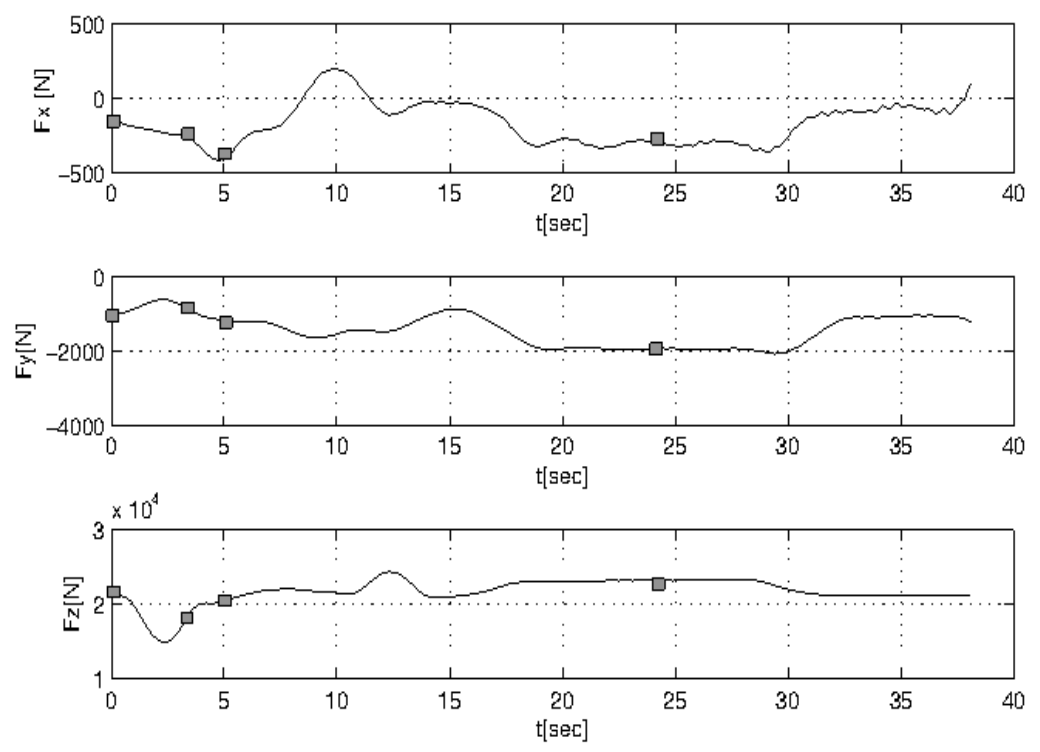

Figure 7: Hub forces time-histories.
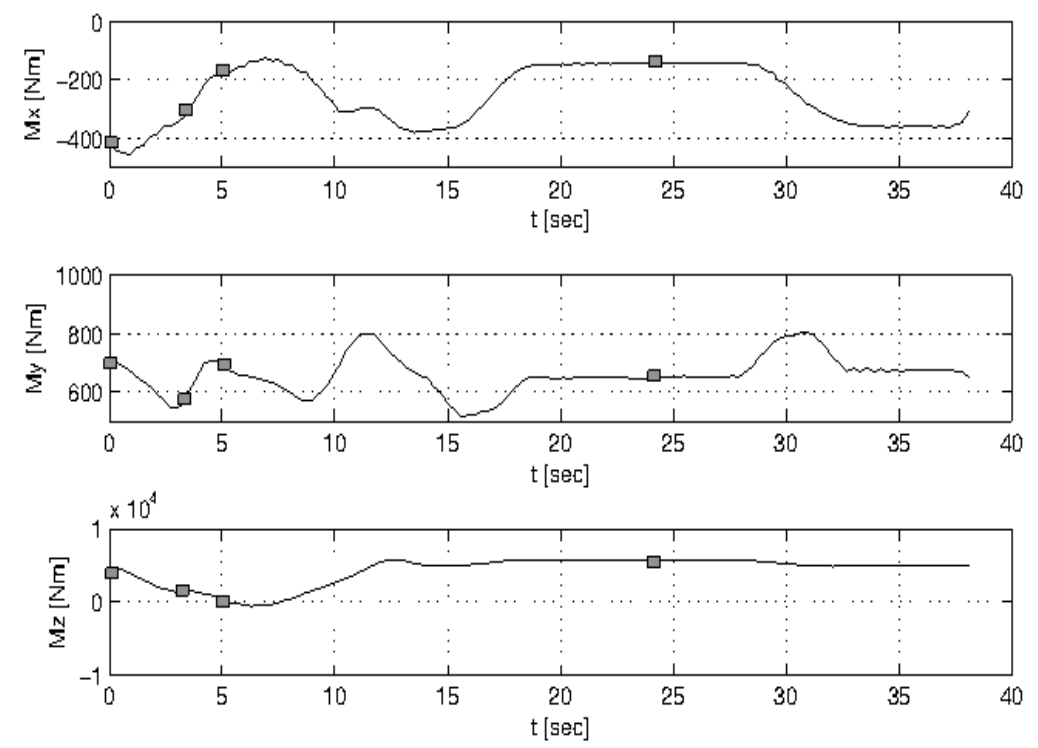

Figure 8: Hub moments time-histories. 


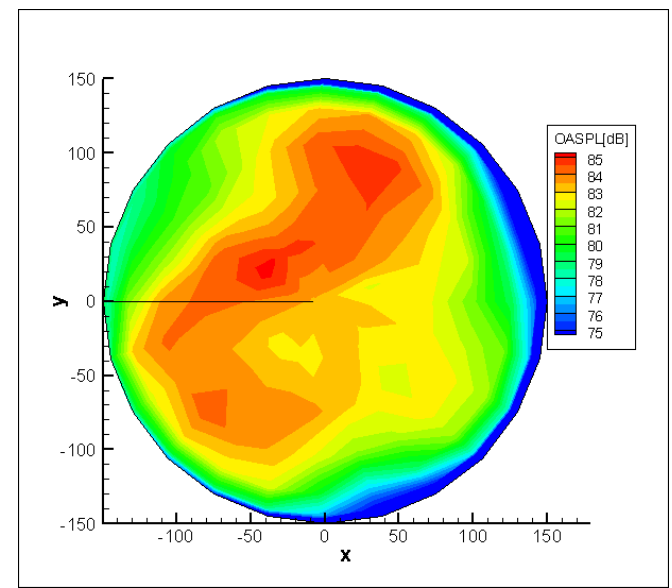

Figure 9: Noise hemisphere at trajectory point 1. 


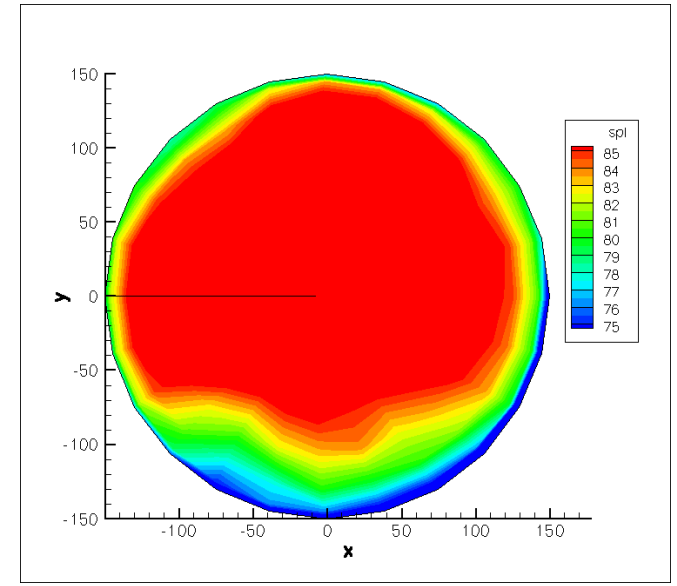

(a) Approach A

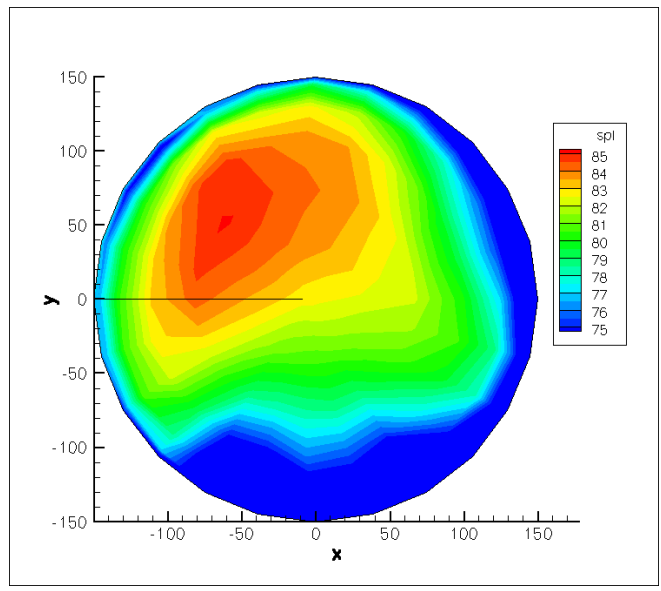

(b) Approach B

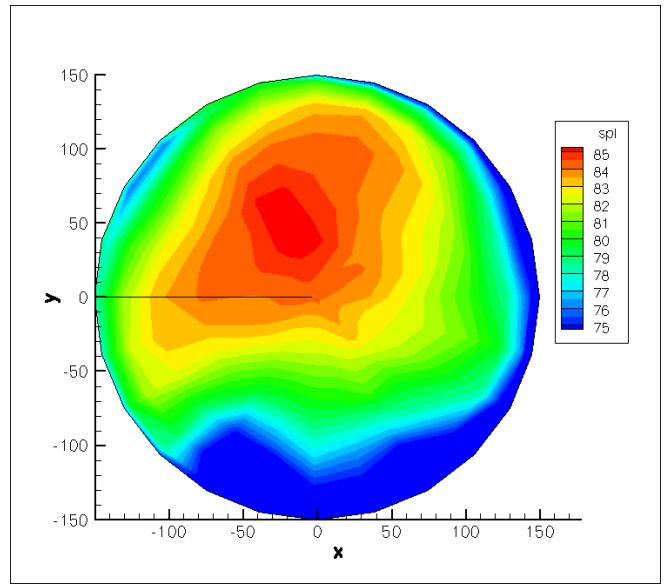

(c) Approach C

Figure 10: Noise hemispheres at trajectory point 2. 


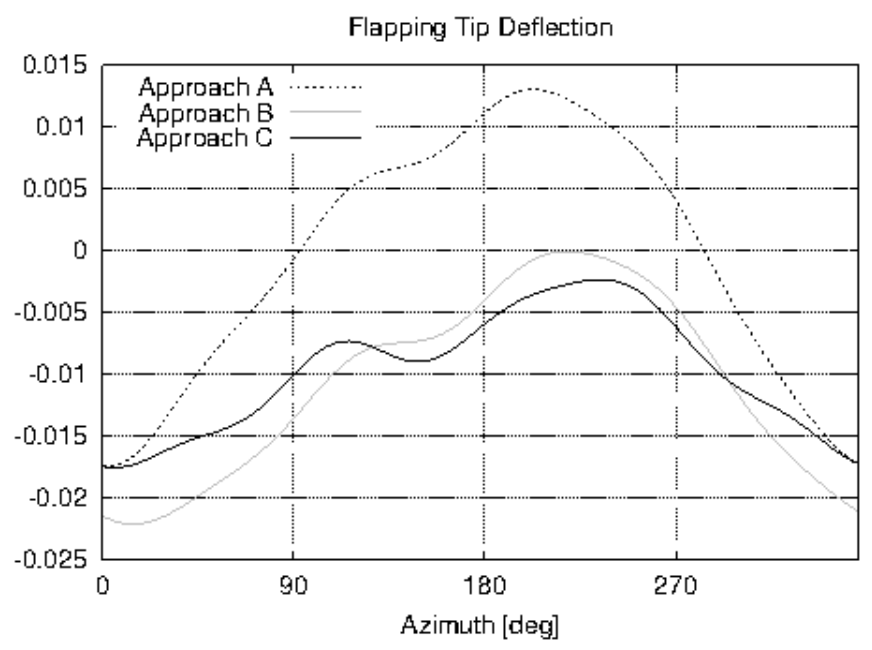

Figure 11: Blade tip flap displacement at trajectory point 2.

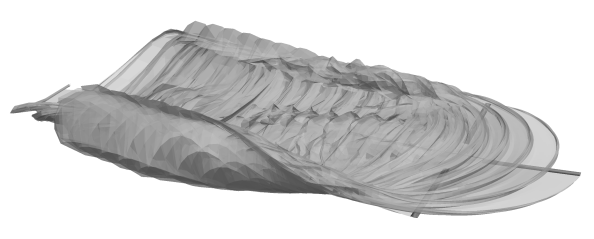

(a) Approach A

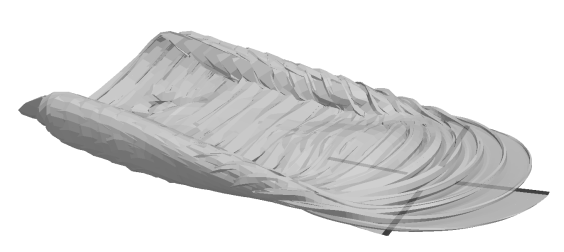

(b) Approach B

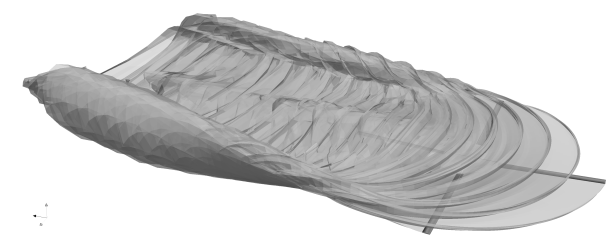

(c) Approach C

Figure 12: Main rotor wake shape at trajectory point 2. 


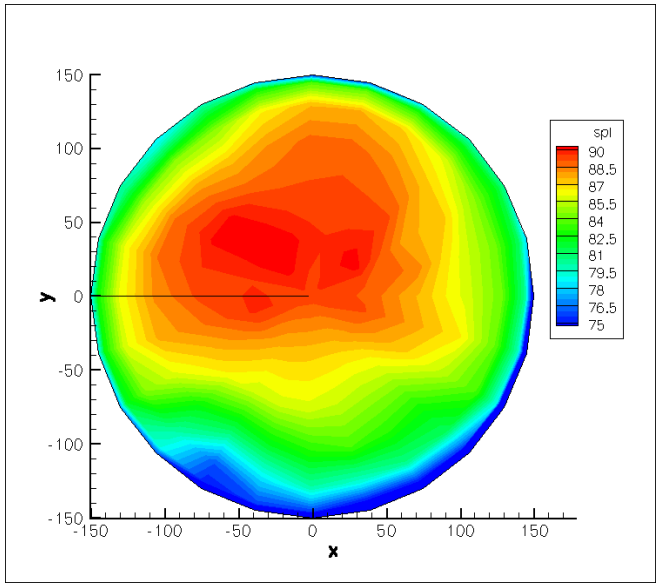

(a) Approach A

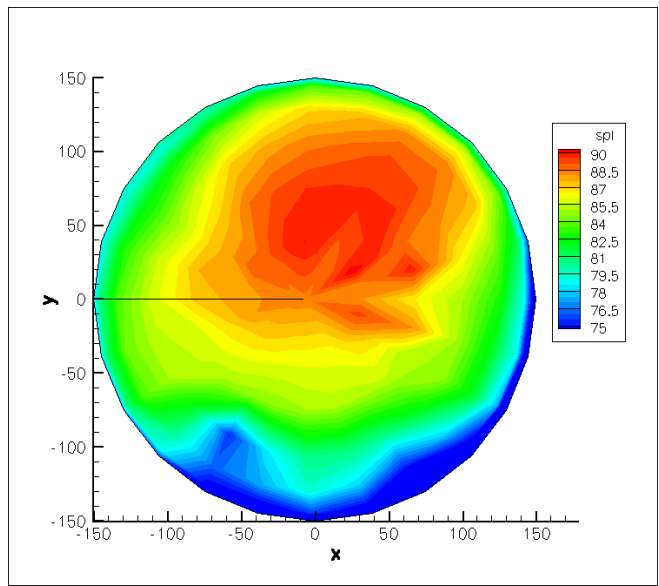

(b) Approach B

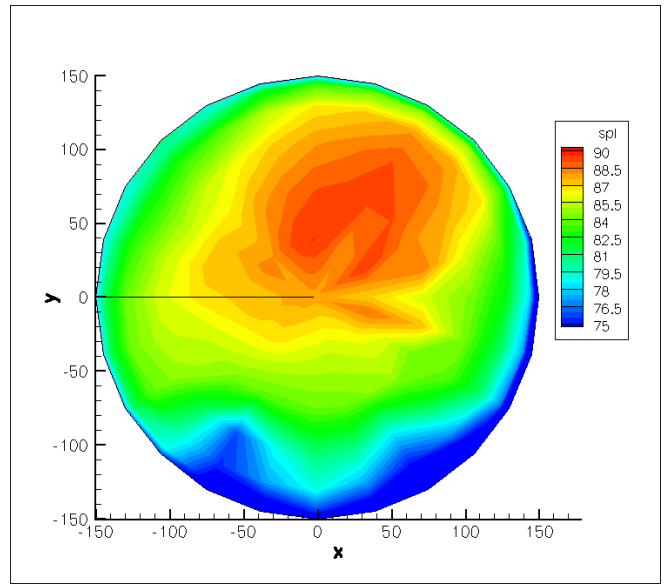

(c) Approach C

Figure 13: Noise hemispheres at trajectory point 3. 


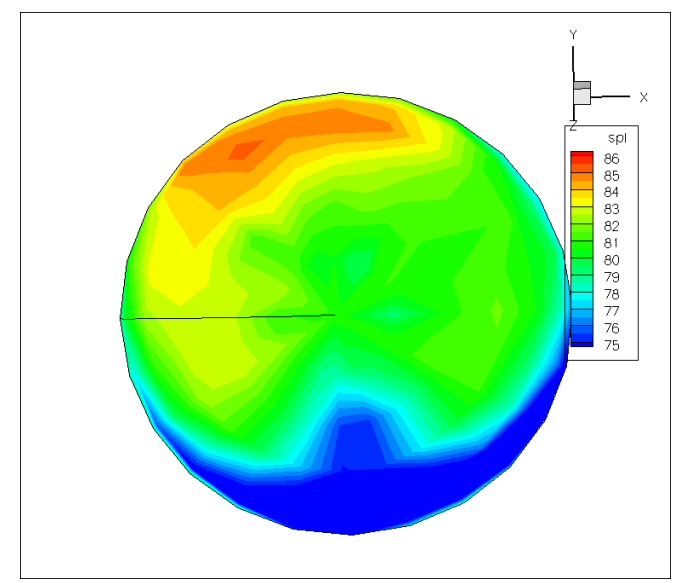

(a) Approach A

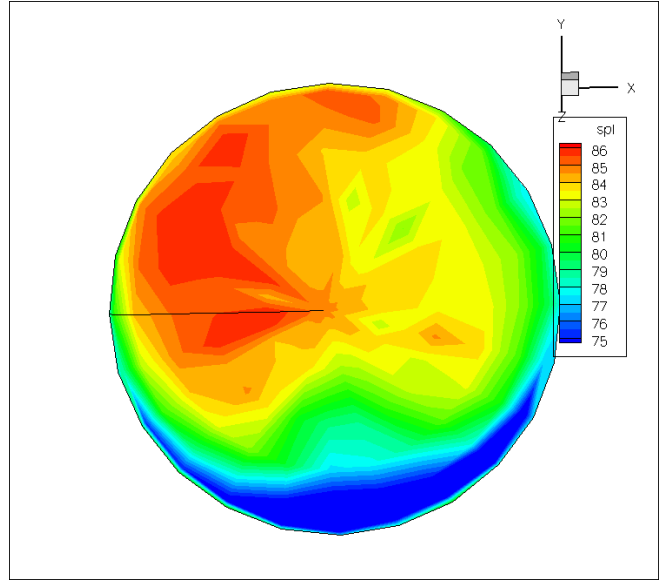

(b) Approach B

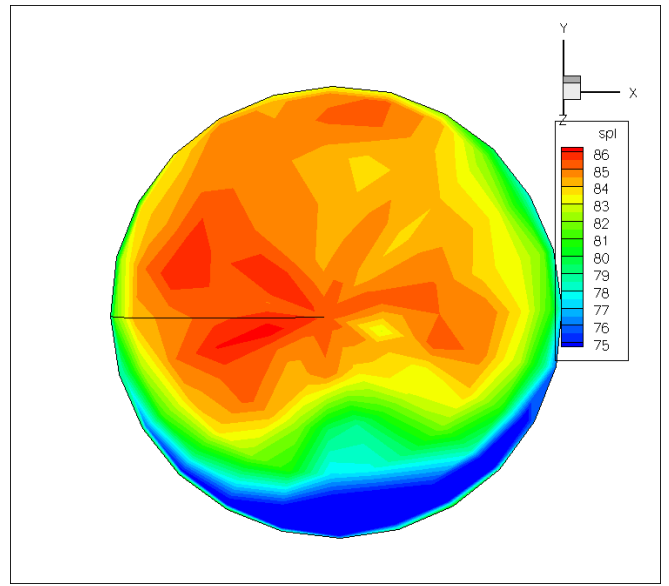

(c) Approach C

Figure 14: Noise hemispheres at trajectory point 4 . 